\title{
Workplace Ostracism: More Distressing than Harassment
}

\author{
${ }^{1}$ Dr. Ruchi Sao, ${ }^{2}$ Dr. Kanak Wadhwani \\ ${ }^{1,2}$ Assistant Professor, Shri Ramdeobaba College of Engineering and Management, Nagpur \\ Email:saorn@rknec.edu,wadhwanik@rknec.edu
}

\author{
Received: $20^{\text {th }}$ September 2018, Accepted: $11^{\text {th }}$ October 2018, Published: $31^{\text {st }}$ October 2018
}

\begin{abstract}
The current study is a qualitative one which uses focus group discussions (FGD) and interviews to understand the existence and instances of workplace ostracism. Literature review including research papers and articles written by HR practitioners has been done. The idea was to understand the various factors that affect the occurrence of ostracism at the workplace.

Workplace ostracism is a process of ostracizing or excluding someone at the workplace on any ground. The research paper presents a qualitative study which uses Focus Group Discussions (FGD) and interviews. The factors causing workplace ostracism are identified through research which are colleagues, self, organization culture, leader and work environment. A questionnaire has been made on the basis of the factors identified and is validated. This questionnaire can be used to identify the factors which contribute towards workplace ostracism. 29 statements have been identified which makes an attempt to identify the existence or nonexistence of workplace ostracism at the workplace. It is developed with the objective to help the organizations to conduct workplace ostracism studies across the employees.
\end{abstract}

\section{Keywords \\ Culture, Social Exclusion, Workplace Ostracism}

\section{Introduction}

If a person does not get a response when he enters a room of people or people take time to reply can be fine for an individual. However, if the same person is treated as if he does not exist would be more killing and distressing for the individual. Sometimes, it is not the physical pain that breaks you down but the mental agony in the form of social exclusion that breaks you down.

There are umpteen examples of presence of ostracism at workplace such as not even considering opinion of juniors or some employees, stop communication when a person enters the room, passing remarks in an arrogant way on purpose to demean a person, going beyond bullying to make the other person feel bad.

Not many of us are with a perfect workplace environment. Ostracism is not practiced but is still faced by many junior and middle level employees in all sectors of business. There are various terms used synonymously with ostracism such as bullying, harassment and sometimes a reason of conflict. Workplace ostracism is defined by Robinson as the degree with which an individual or group omits another organizational member from engaging in socially appropriate actions. Types of behavior that leads to ostracism range from minor exclusionary tactics such as curt responses to more serious instances, with the most serious form of ostracism involving death (Gruter, Et al 1986).

Some of the critical incidents reported on the similar line are exclusion from formal or informal conversations, leaving the area when someone enters, feeling isolated and alone, and feeling of being invisible at workplace for others, no positive interpersonal communication and many more.

When employees feel excluded on the basis of their own perception it is linked to high social anxiety (Baumeiste, Et al, 1990), anger (Twenge, Et al, 2001), hurt feelings (Leary, Et al, 1998), loneliness (Jones, 1990) and lower psychological health (Schneider et al., 2000).

Ostracism behaviors may take any form which can include giving silent treatment to others, no response attitude, being excluded or ignoring someone beyond an extent to make the person upset or even outright rejection.

The study involves qualitative techniques; focus group discussions (FGD) and interviews with employees across hierarchy in public sector undertakings (PSU) sector in India to understand factors that contribute towards workplace ostracism. Four FGD's and seven interviews were taken across hierarchy; factors were identified contributing to workplace ostracism which are colleagues, self, organization culture, leader and work environment. Workplace harassment can actually give more stress as compared to other forms of mental harassment.

A questionnaire has been made on the basis of the factors identified and is validated. This questionnaire can be used to identify the factors which contribute towards workplace ostracism. 
The FGD were conducted by contacting officials working in different PSU's which included banking, power and mining sector. The authors acted as moderator in the FGD.

All members involved in the FGD were well informed about the purpose of the discussion. All the groups comprised of both male and females and were brought together to bring opinions without hesitation. The members of each FGD were belonging to all age groups to avoid homogeneity during discussions.

\section{Material and Methods}

Colleagues, self, organization culture, leader and work environment are the factors which were studied and were found to be practically applicable when it came to practice by many people responding to the interviews. The following statements are included in the questionnaire and the respondents can rate it on a scale of strongly disagree to strongly agree.

- It is easy for me to initiate conversation with my colleagues.

- I feel out of place when I am with my colleagues in the office.

- I feel left out during the discussions with my colleagues.

- I feel free to discuss work related problems with my fellow colleagues.

- My colleagues are always open to offer help without discussing it with others.

- I feel that I am misunderstood by my peer at office.

- I feel scared to express my views before my seniors.

- I need support and guidance from my team leader / supervisor.

- I receive support and guidance from my team leader / supervisor.

- I fear being fired or being scolded for my work or decisions.

- I feel stressed when I am called to report my team leader / supervisor.

- I feel my greetings are unanswered by my colleagues.

- I often feel that employees ignore me at workplace.

- When I enter office or any other area in office (for eg., parking space) or outside office; other people start leaving after seeing me.

- When employees see me coming, they stop talking in the group.

- You are often forced to sit alone during meals and breaks.

- I feel that I am treated as if I am not present only.

- I feel discomfort working due to unhealthy working environment.

- I feel stressed and heavy from within when I enter the workplace.

- I feel low when I think of going to office.

- I feel sad or depressed if even a small thing goes wrong.'

- I feel positive about the working culture of the organization.

- Interpersonal relations are good at my workplace.

- Even when people are not ignoring me, I still feel that I am isolated.

- If you have worked in other organizations earlier, you faced similar problem of exclusion from colleagues.

- I am rigid to my way of working and avoid other's interference.

- I feel nervous or anxious about my feedback from my supervisor.

- I wish to quit the job due to work pressure.

- I often take jokes or funny talks personally.

\section{Results and Discussion}

Total four FGD's were conducted in which the employees opened up by sharing their stories of early joining. Even today, many employees have to do the work which is not even directly or indirectly related to their job descriptions. All the findings of FGD have been merged together and has been reported as under:

- The employees who have confidence, do not fall prey to workplace ostracism. These are the employees who have a strong body language, high self-esteem and have positive beliefs.

- Employees coming from small cities are generally brought up in a family kind of environment and the moral and value system is high. These kind of employees often receive a lot of care and affection throughout their childhood and adulthood. Some members of FGD prominently noted that these employees are hardworking and feel guilty if they are unable to complete their tasks on time. These feelings sometimes make them vulnerable to people who believe in ostracizing someone from workplace or an informal circle. 
- Communication also acts as a factor for workplace ostracism. Employees with low communication or not having proper voice accent or command over English language, may have feelings of ostracism. Often, other employees who are better in terms of communication and like mindedness with such people may have these kind of feelings very easily.

- The culture of the organization plays the most important role. Example of IT Company was quoted where the hiring, appraisals and all other activities are purely on the basis of merit. This means that tall the employees are aware about their own performance. Unlike in PSU sector, all the employees are given annual increments which are similar in nature. The culture needs to have positive competition. It helps all employees to focus on their work not resulting into ostracism.

- Sometimes the culture of the organization is really good. Whereas, a team leader can influence the mini culture of the team. If the team leader decides to play the game in the team, more mini groups will be created resulting into unhealthy environment. In such a case, team members can become biased and may involve themselves into ostracism.

- Workplace ostracism is not always intentional in nature, there are many instances in which the employee himself may feel that he is been ostracized. This may be either because of some wrong done by him or the feeling of being incompetent to be a part of the group.

Interviews were taken from people working in PSU sector. Total seven interviews were taken. The interviewees were taken from across the hierarchy which resulted into a lot of information collection. Interviews are reported as under:

- Feeling of ostracism may be self-inflicted also. For instance, when a person reaches and the group disperses, it does not mean that they are ostracizing someone. The reason may also be that the group has already finished their discussion. However, if the same instance may keep on getting repeated with the same person, it can definitely be interpreted that it is workplace ostracism with such an individual.

- The manner in which one looks and presents oneself may also be a decisive factor. A person having a good personality in terms of communication, looks and otherwise, may not be a victim to ostracism. Of course, people may talk behind him. But, that individual will never be ostracized.

- It is also related to the work pressures, stress and anxiety with respect to individual employees.

- Workplace ostracism is more of a feeling. If the employees decide to ignore and get affected by it, there will not be any kind of ostracism to such a person.

- Ostracism is a feeling of being ignored which may get negative for the person and would affect his performance and productivity levels.

- Workplace ostracism has also been termed by many as cold violence in which the injury is only on mind and later affecting the performance of the person.

- The concept of ostracism varies from an individual to another. It is not necessary that if one person is feeling ostracized, everyone would feel the same.

- Ostracism may take the form of social rejection which may have a lifelong impact on the mental wellbeing of the employee.

Thus, it is important that the overall culture should be conducive enough for the psychological wellbeing and growth of the individual.

The questionnaire has been developed through this qualitative research and literature review. Through the use of questionnaire, companies can identify the factors causing workplace ostracism and can work on the eradication of the same. Ostracism is a painful process which causes a sense of social pain and decreases the morale of such an employee.

The outcome of the study is the questionnaire which is given as under.

\section{Conclusion}

The present study identifies the common factors causing workplace ostracism. The findings are arrived at through FGD's and interviews of various employees working across the hierarchy in PSU sector. The study will contribute to the field of literature in the area of workplace ostracism in PSU sector in India. In practice, the companies can use the questionnaire developed to remove barriers within the workplace by addressing the issues on ostracism. The statements can be used with demographic data to check the level of workplace ostracism in any organization.

The occurrence of workplace ostracism leads to fall in efficiency of the employee and low sense of belongingness. Ostracized employees has high probability of leaving the organization or staying and remain emotionally and 
mentally absent from work. The employee commitment level would also go down which can be detrimental for the organization at large. Workplace ostracism may turn out to be more stressing as compared to harassment.

Thus, it is important for the leaders to create a working environment which is conducive and friendly for all the employees. The organizations should have an open communication system where the employees can speak their heart. There should be regular coaching and mentoring sessions which help the employees to speak up.

\section{References}

1. Robinson, S.L., O'Reilly, J. and Wang, W., 2013. Invisible at work: An integrated model of workplace ostracism. Journal of Management, 39(1), pp.203-231

2. Gruter, M. and Masters, R.D., 1986. Ostracism as a social and biological phenomenon: An introduction

3. Baumeister, R.F. and Tice, D.M., 1990. Point-counterpoints: Anxiety and social exclusion. Journal of social and clinical Psychology, 9(2), pp.165-195

4. Jones, W.H., 1990. Loneliness and social exclusion. Journal of Social and Clinical Psychology, 9(2), pp.214220

5. Twenge, J.M., Baumeister, R.F., Tice, D.M. and Stucke, T.S., 2001. If you can't join them, beat them: effects of social exclusion on aggressive behavior. Journal of personality and social psychology, 81(6), p.1058

6. Leary, M.R., Springer, C., Negel, L., Ansell, E. and Evans, K., 1998. The causes, phenomenology, and consequences of hurt feelings. Journal of personality and social psychology, 74(5), p.1225

7. Schneider, K.T., Hitlan, R.T. and Radhakrishnan, P., 2000. An examination of the nature and correlates of ethnic harassment experiences in multiple contexts. Journal of Applied Psychology, 85(1), p.3 